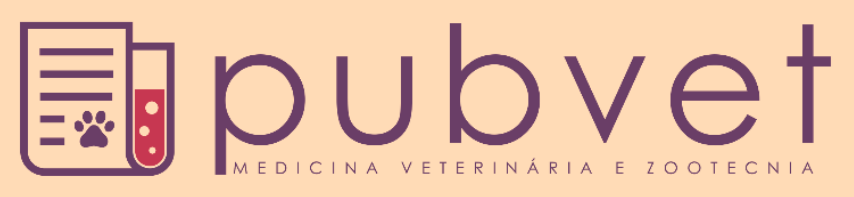

https://doi.org/10.31533/pubvet.v12n12a230.1-10

\title{
Produção e composição química de variedades de cana-de-açúcar com fins forrageiros sob irrigação e adubação
}

\author{
João Avelar Magalhães ${ }^{1 \oplus}$, Braz Henrique Nunes Rodrigues $^{1 \oplus}$, Francisco José de Seixas Santos ${ }^{1} \bullet$, \\ Aderson Soares Andrade Junior ${ }^{2} \bullet$, Raimundo Bezerra de Araújo Neto ${ }^{2}$, Newton de Lucena \\ $\operatorname{Costa}^{30}$, Danielle Maria Machado Ribeiro Azevêdo ${ }^{2}{ }^{\ominus}$, Roberto Claudio Fernandes Franco \\ Pompeu $^{4}$, Karina Neoob de Carvalho Castro $^{1} \bullet$
}

${ }^{1}$ Pesquisador da Embrapa Meio-Norte. Parnaíba, PI.
${ }^{2}$ Pesquisador da Embrapa Meio-Norte. Teresina, PI.
${ }_{3}^{3}$ Pesquisador da Embrapa Roraima. Boa Vista, RR.
${ }^{4}$ Pesquisador Embrapa Caprinos e Ovinos. Sobral, CE
${ }^{*}$ Autor para correspondência, E-mail: joao.magalhaes@embrapa.br

RESUMO. Objetivou-se avaliar os efeitos de variedades, da irrigação e da adubação sobre a produção e composição química da cana-de-açúcar com fins forrageiros em Parnaíba, Piauí. O delineamento experimental foi em blocos casualizados, em esquema fatorial $2 \times 5 \times 4$, com quatro repetições. Os tratamentos consistiram em duas lâminas de irrigação (reposição de $50 \%$ e $100 \%$ da evapotranspiração de referência - ETo), cinco variedades de cana-de-açúcar (RB962962, RB867515, RB92579, RB863129 e RB98710) e quatro níveis de adubação $(70+63 ; 140+126 ; 210+189$ e $280+252 \mathrm{~kg}$ de $\mathrm{N}+$ $\mathrm{K}_{2} \mathrm{O} / \mathrm{ha} / \mathrm{ano}$, respectivamente). O plantio foi realizado em julho de 2015 e a colheita em julho de 2016. As variedades de cana-de-açúcar RB962962 e RB867515 se sobressaíram das demais, apresentando elevada produtividade de biomassa e maiores percentuais de colmos, conciliada a satisfatórios teores de sólidos solúveis (açúcares). Para as condições do norte do Piauí, considerando-se a produtividade, melhoria da composição química e a possibilidade de economia de água, a lâmina de água aplicada (lâmina de irrigação + $1.696 \mathrm{~mm}$ de precipitação pluviométrica), associada à aplicação de $70 \mathrm{~kg}$ de $\mathrm{N}$ e $63 \mathrm{~kg}$ de $\mathrm{K}_{2} \mathrm{O} / \mathrm{ha} / \mathrm{ano}$, pode ser utilizada na produção de biomassa da cana-de-açúcar com fins forrageiros.

Palavras-chave: Brix, fibra, morfometria, produtividade, proteína bruta, Saccharum officinarum

\section{Yield and chemical composition of sugarcane forage varieties under irrigation and fertilization}

ABSTRACT. The effects of varieties, irrigation and fertilization on the production and chemical composition of sugarcane with forage purposes were evaluated in Parnaíba, Piauí. The experimental design was in randomized blocks, in a $2 \times 5 \times 4$ factorial scheme, with four replications. The treatments consisted of two irrigation depths $(50 \%$ and $100 \%$ of the reference evapotranspiration - ETo), five sugarcane varieties (RB962962, RB867515, RB92579, RB863129 and RB98710) and four doses of fertilization (70 + 63; $140+126 ; 210+189$ and $280+252 \mathrm{~kg}$ of $\mathrm{N}+\mathrm{K}_{2} \mathrm{O} / \mathrm{ha} /$ year, respectively). The planting was carried out in July 2015 and the harvest in July 2016. The sugarcane varieties RB962962 and RB867515 stood out from the others, presenting high productivity of biomass and higher percentage of stalks, reconciled with satisfactory levels of soluble solids (sugars). For the northern Piauí conditions, considering the productivity, improvement of the chemical composition and the possibility of water saving, the applied 
water depth (irrigation depth $+1,696 \mathrm{~mm}$ of rainfall), associated with the application of $70 \mathrm{~kg}$ of $\mathrm{N}$ and $63 \mathrm{~kg}$ of $\mathrm{K}_{2} \mathrm{O} / \mathrm{ha} /$ year, can be used in the production of biomass of sugarcane for fodder purposes.

Key Words: Brix, fiber, morphometry: productivity, crude protein, Saccharum officinarum

\title{
Producción y composición química de variedades de caña de azúcar con fines forrajeros bajo riego y fertilización
}

\begin{abstract}
RESUMEN. Se pretendió evaluar los efectos de variedades, de la irrigación y de la fertilización sobre la producción y composición química de la caña de azúcar con fines forrajeros en Parnaíba, Piaú. El delineamiento experimental fue en bloques casualizados, en esquema factorial $2 \times 5 \times 4$, con cuatro repeticiones. Los tratamientos consistieron en dos láminas de irrigación (reposición del $50 \%$ y $100 \%$ de la evapotranspiración de referencia - ETo), cinco variedades de caña de azúcar (RB962962, RB867515, RB92579, RB863129 y RB98710) y cuatro niveles de fertilización $(70+63 ; 140+126 ; 210+189$ y $280+252 \mathrm{~kg}$ de $\mathrm{N}+\mathrm{K}_{2} \mathrm{O} / \mathrm{ha} /$ año, respectivamente). La siembra fue realizada en Julio de 2015 y la cosecha en Julio de 2016. Las variedades de caña de azúcar RB962962 y RB867515 se sobresalieron de las demás, presentando elevada productividad de biomasa y mayores porcentajes de colmos, concilia a satisfactorios contenidos de sólidos solubles (azúcares). Para las condiciones del norte de Piauí, considerando la productividad, mejora de la composición química y la posibilidad de ahorro de agua, la lámina de agua aplicada (lámina de irrigación $+1.696 \mathrm{~mm}$ de precipitación pluviométrica), asociada a la aplicación de $70 \mathrm{~kg}$ de $\mathrm{N}$ y $63 \mathrm{~kg}$ de $\mathrm{K}_{2} \mathrm{O} / \mathrm{ha} / \mathrm{año}$, puede utilizarse en la producción de biomasa de la caña de azúcar con fines forrajeros.
\end{abstract}

Palabras clave: Brix, fibra, morfometría, productividad, proteína bruta, Saccharum officinarum

\section{Introdução}

Com um rebanho superior a 49 milhões de animais, formado de pequenos e grandes ruminantes, a pecuária é uma das atividades econômicas mais importantes da região Nordeste (ANUALPEC 2018). Contudo, a região é caracterizada por índices pluviométricos irregulares com amplos períodos de seca e má distribuição de chuvas, principalmente no Bioma Caatinga, que possui características semiáridas e corresponde a 54\% da área total (Andrade et al. 2010).

Neste cenário a cana-de-açúcar (Saccharum officinarum) surge como uma das opções para assegurar melhor padrão alimentar dos animais durante os períodos críticos de escassez de forragem, pois fisiologicamente, ao contrário das demais gramíneas tropicais, seu valor nutritivo se mantém praticamente inalterado com a maturidade, apesar do baixo nível de proteína bruta (Fernandes et al. 2003). Essa característica é justificada pelo incremento nos teores de açucares, carboidrato de alta digestibilidade ao mesmo tempo em que ocorre redução na concentração de componentes da parede celular, que apresentam baixa digestibilidade (Andrade et al. 2004; Carvalho et al. 2010). É relativamente fácil de ser implantada e manejada, podendo atingir, quando irrigada e adubada, rendimentos superiores a 250 toneladas de massa verde/ha/ano (Pigden 1974; Oliveira et al. 2002; Oliveira et al. $\underline{2015)}$.

Cândido et al. (2005) e Santos et al. (2011) consideram a cana-de-açúcar uma forrageira não convencional, perfeitamente adaptada às condições do semiárido. A cana-de-açúcar pode ser cultivada com e sem irrigação nos brejos de altitude, nos vales, em terras de várzea e margeando os cursos d'água, além dos perímetros irrigados (Carlos Filho et al. 1986; Braga et al. 2002; Nunes Filho et al. 2007; Voltolini et al. 2012). No Brasil, a produção da cana-de-açúcar é destinada, principalmente, à indústria sucroalcooleira. No entanto, Landell et al. (2002) e Freitas et al. (2011) estimam que $10 \%$ de sua produção são utilizadas na alimentação de ruminantes.

Os trabalhos iniciais com melhoramento de cana-de-açúcar destinada à alimentação animal 
visavam principalmente ao aumento no valor proteico desta forrageira (Bonomo et al. 2009). Atualmente os critérios para escolha de uma variedade de cana-de-açúcar como forrageira tem-se baseado, além da produtividade, nos teores de FDN (fibra em detergente neutro), nos teores de açúcares (Brix) e na razão FDN/Brix. A razão FDN/Brix proposta por Rodrigues et al. (1997) considera o teor de fibra em relação ao teor de sólidos solúveis da cana-de-açúcar; e quanto mais baixa a razão, melhor seu valor como alimento para bovinos. Ademais, Rodrigues et al. (1997) observaram que, quanto menor a razão FDN/açucares, maior será a digestibilidade in vitro da matéria seca.

Por outro lado, a cana-de-açúcar apresenta elevado consumo de água. Cintra et al. (2008) reportaram que durante o ciclo a cana-de-açúcar pode consumir até $4,74 \mathrm{~mm}$ de água/dia. Para o crescimento e desenvolvimento potencial da cana ser atingido, a mesma necessita de $1.500 \mathrm{~mm}$ a $2.500 \mathrm{~mm}$ anuais (Doorembos \& Kassam 1979). Contudo, apenas a utilização de novas variedades e o uso da irrigação pode não ser suficiente para melhorar os índices produtivos da cana-de-açúcar para alimentação animal, sendo necessário o uso da adubação, principalmente com nitrogênio e potássio, macro nutriente, mais demandado pela cana-de-açúcar. A irrigação associada a adubação nitrogenada e potássica tem promovido incremento na produção da cana-de-açúcar (Dalri \& Cruz 2008), podendo superar $200 \mathrm{t} / \mathrm{ha}$.

O objetivo deste trabalho foi avaliar os efeitos de lâminas de irrigação, de variedades e da adubação sobre a produção e composição química da cana-de-açúcar com fins forrageiros.

\section{Material e métodos}

O experimento foi conduzido durante o período de 12 meses, decorridos entre 3 julho de 2015 a 4 de julho de 2016, na Unidade de Execução de Pesquisa de Parnaíba, pertencente à Embrapa Meio-Norte, com as seguintes coordenadas geográficas, $3^{\circ} 5^{\prime}$ de latitude sul, $41^{\circ}$ $46^{\prime}$ de longitude oeste e $46,8 \mathrm{~m}$ altitude. O clima da região, segundo Köppen and Geiger (1928) é Aw, com estação seca bem definida (julho a dezembro) e pluviosidade média anual de 1.300 $\mathrm{mm}$. O solo da área experimental é da classe Latossolo Amarelo distrófico, textura média, fase caatinga litorânea e relevo plano e suave ondulado (Melo et al. 2004). No início do experimento o solo apresentou as seguintes características químicas: $\mathrm{MO}=1,34 \mathrm{dag} / \mathrm{kg} ; \mathrm{pH}$ $\left(\mathrm{H}_{2} \mathrm{O}\right)=5,79 ; \mathrm{P}=23 \mathrm{mg} / \mathrm{dm}^{3} ; \mathrm{N}=0,04 \mathrm{dag} / \mathrm{kg}$; $\mathrm{K}=0,21 \mathrm{cmol}_{\mathrm{c}} / \mathrm{dm}^{3} ; \mathrm{Ca}=2,68 \mathrm{cmol}_{\mathrm{c}} / \mathrm{dm}^{3} ; \mathrm{Mg}=$ $0,38 \mathrm{cmol}_{\mathrm{d}} / \mathrm{dm}^{3} ; \mathrm{Na}=0,09 \mathrm{cmol}_{\mathrm{c}} / \mathrm{dm}^{3} ; \mathrm{Al}=0,05$ $\mathrm{cmol}_{\mathrm{d}} / \mathrm{dm}^{3} ; \mathrm{H}+\mathrm{Al}=2,60 \mathrm{cmol}_{\mathrm{c}} / \mathrm{dm}^{3} ; \mathrm{SB}=2,14$ $\mathrm{cmol}_{\mathrm{C}} / \mathrm{dm}^{3} ; \quad$ CTC $=5,85 \quad \mathrm{cmol}_{\mathrm{d}} / \mathrm{dm}^{3}$, respectivamente.

$\mathrm{O}$ delineamento experimental foi em blocos casualizados, em esquema fatorial 2 × 5 x 4, com quatro repetições. Os tratamentos consistiram em duas lâminas de irrigação (reposição de $50 \%$ e $100 \%$ da evapotranspiração de referência - ETo), cinco variedades de cana-de-açúcar (RB962962, RB867515, RB92579, RB863129 e RB98710) e quatro níveis de adubação $(70+63 ; 140+126$; $210+189$ e $280+252 \mathrm{~kg}$ de N $\left.+\mathrm{K}_{2} \mathrm{O} / \mathrm{ha} / \mathrm{ano}\right)$.

Em julho de 2015, realizou-se o plantio em solo arado, gradeado e sulcado no espaçamento $1,2 \mathrm{~m} \times 0,30 \mathrm{~m}$. Os colmos foram distribuídos nos sulcos numa densidade de 12 a 18 gemas $/ \mathrm{m}$ linear. No plantio, foram distribuídos em fundação $100 \mathrm{~kg} / \mathrm{ha}$ de $\mathrm{P}_{2} \mathrm{O}_{5}$ (superfosfato triplo), para todos os tratamentos. As doses de NK foram fracionadas, obedecendo aos tratamentos, em três aplicações: $30 \%$ aos 15 dias pós-plantio $(21 \mathrm{~N}+$ $18,9 \mathrm{~K} ; 42 \mathrm{~N}+37,8 \mathrm{~K} ; 63,0 \mathrm{~N}+56,7 \mathrm{~K} 84,0 \mathrm{~N}+$ $75,6 \mathrm{~K}) ; 35 \%$ aos 60 dias pós-plantio $(24,5 \mathrm{~N}+$ $22,05 \mathrm{~K} ; 49,0 \mathrm{~N}+44,10 \mathrm{~K} ; 73,5 \mathrm{~N}+66,15 \mathrm{~K}$ e $98,0 \mathrm{~N}+88,20 \mathrm{~K})$ e $35 \%$ aos 90 dias pós-plantio $(24,5 \mathrm{~N}+22,05 \mathrm{~K} ; 49,0 \mathrm{~N}+44,10 \mathrm{~K} ; 73,5 \mathrm{~N}+$ $66,15 \mathrm{~K}$ e $98,0 \mathrm{~N}+88,20 \mathrm{~K})$.

Quanto a irrigação, foi utilizado o sistema com fita gotejadora com turno de rega de dois dias. Os valores das lâminas totais de irrigação aplicadas nos tratamentos dos níveis de irrigação foram 890,6 mm (50\% ETo) e 1129,61 mm (100\% ETo). Durante o período experimental a precipitação pluviométrica foi de $805,6 \mathrm{~mm}$, assim as lâminas totais aplicadas, (lâminas de irrigação + pluviosidade) foram de 1696,20 mm e 1935,21 mm, respectivamente, para os tratamentos de $50 \%$ ETo e $100 \%$ ETo. Ressaltase que os tratamentos diferenciados de irrigação só se iniciaram aos 111dias de implantação da cultura, quando até então, foram aplicadas as lâminas correspondentes a 100\% de Eto para ambos os tratamentos, sob pena da cultura não se desenvolver no menor aporte de água. Dessa forma, a diferença entre o tratamento de $50 \%$ de ETo foi de 239,01 mm, considerando os 111 dias de uniformização das lâminas e o total de precipitação pluviométrica que foram comuns aos dois tratamentos. 
O corte foi realizado manualmente em julho de 2016, quando foram averiguados os seguintes parâmetros: altura de planta e tamanho do colmo, produtividade total de matéria verde (PTMV) e produtividade de matéria verde de colmos (PMVC). Para determinação do tamanho, diâmetro e percentual de colmos e da produção de matéria verde dos mesmos, a planta inteira foi separada em ponteira e colmo. A concentração de sólidos solúveis (Brix) foi aferida em amostras de caldo extraído de três colmos, utilizando-se refratômetro manual de alto contraste.

Para a estimativa da produtividade total de matéria seca (PTMS) e da composição química, foram obtidas amostras que foram trituradas e pesadas em balança eletrônica, e submetidas à pré-secagem em estufa com circulação forçada de ar, a $65^{\circ} \mathrm{C}$ até peso constante. No material colhido foi determinado os teores de proteína bruta (PB), pelo método de Kjeldahl, fibra em detergente neutro (FDN) e fibra em detergente ácido (FDA) de acordo com a metodologia preconizada por Silva and Queiroz (2002). A digestão para obtenção da FDN e FDA foi realizada em aparelho de digestão de fibra. As amostras foram colocadas em sachê de tecido de TNT $100 \mathrm{~mm}$, sendo que em cada um foi depositado $0,5 \mathrm{~g}$ de massa da cana-de-açúcar préseca moída em peneira com malha de $1 \mathrm{~mm}$.

Os resultados foram submetidos à análise de variância para observação da existência ou não de interação lâminas de irrigação $\mathrm{x}$ variedades $\mathrm{x}$ adubação e as médias foram comparadas pelo teste de Tukey, ao nível de 5\% de probabilidade. Os dados foram analisados utilizando-se o programa estatístico Assistat (Silva and Azevedo 2016).

\section{Resultados e discussão}

Os efeitos isolados das lâminas de irrigação sobre a altura de plantas, tamanho e diâmetro do colmo da cana-de-açúcar, bem como a interação entre lâminas de irrigação, variedades e níveis de adubação não foram significativos $(\mathrm{P}>0,05)$, revelando assim que, para a obtenção da máxima altura de plantas, tamanho e diâmetro do colmo, torna-se dispensável a reposição total da evapotranspiração. Independentemente da variedade e da adubação, a cana-de-açúcar se desenvolveu bem sob a menor lâmina de água de irrigação, chegando a atingir valores médios de 4,33 $\mathrm{m}$ de altura de plantas, 3,26 $\mathrm{m}$ de tamanho do colmo e $27,4 \mathrm{~mm}$ de diâmetro de colmos (Tabela 1).

Tabela 1. Respostas da altura de plantas (AP), do tamanho do colmo (TC) e diâmetro do colmo (DC) da cana-de-açúcar aos efeitos isolados das lâminas de irrigação, variedades e adubação. Parnaíba-PI, 2016.

\begin{tabular}{|c|c|c|c|c|c|}
\hline Lâminas de irrigação & $\mathrm{AP}(\mathrm{m})$ & $\begin{array}{l}\text { Variedades cana-de- } \\
\text { açúcar }\end{array}$ & $\mathrm{AP}(\mathrm{m})$ & $\begin{array}{c}\text { Adubação } \\
(\mathrm{kg} / \mathrm{ha})\end{array}$ & $\mathrm{AP}(\mathrm{m})$ \\
\hline \multirow{3}{*}{$1696,20 \mathrm{~mm}$} & \multirow{3}{*}{$4,33 \mathrm{a}$} & RB962962 & $4,27 \mathrm{~b}$ & $70 N+63 \mathrm{~K}$ & $4,42 \mathrm{a}$ \\
\hline & & RB867515 & $4,66 \mathrm{a}$ & $140 \mathrm{~N}+126 \mathrm{~K}$ & $4,29 \mathrm{a}$ \\
\hline & & RB92579 & $4,39 \mathrm{ab}$ & $210 \mathrm{~N}+189 \mathrm{~K}$ & $4,37 \mathrm{a}$ \\
\hline \multirow{2}{*}{$1935,21 \mathrm{~mm}$} & \multirow{2}{*}{$4,41 \mathrm{a}$} & RB863129 & $4,37 \mathrm{ab}$ & $280 \mathrm{~N}+252 \mathrm{~K}$ & $4,40 \mathrm{a}$ \\
\hline & & RB98710 & $4,15 \mathrm{~b}$ & & \\
\hline & $\mathrm{TC}(\mathrm{m})$ & & $\mathrm{TC}(\mathrm{m})$ & & $\mathrm{TC}(\mathrm{m})$ \\
\hline \multirow{3}{*}{$1696,20 \mathrm{~mm}$} & \multirow{3}{*}{$3,26 \mathrm{a}$} & RB962962 & $3,35 \mathrm{ab}$ & $70 \mathrm{~N}+63 \mathrm{~K}$ & $3,32 \mathrm{a}$ \\
\hline & & RB867515 & $3,56 \mathrm{a}$ & $140 \mathrm{~N}+126 \mathrm{~K}$ & $3,31 \mathrm{a}$ \\
\hline & & RB92579 & $3,42 \mathrm{ab}$ & $210 \mathrm{~N}+189 \mathrm{~K}$ & $3,45 \mathrm{a}$ \\
\hline \multirow{3}{*}{$1935,21 \mathrm{~mm}$} & \multirow{2}{*}{$3,48 \mathrm{a}$} & RB863129 & $3,44 \mathrm{ab}$ & $280 \mathrm{~N}+252 \mathrm{~K}$ & $3,41 \mathrm{a}$ \\
\hline & & RB98710 & $3,08 \mathrm{~b}$ & & \\
\hline & $\mathrm{DC}(\mathrm{mm})$ & & $\mathrm{DC}(\mathrm{mm})$ & & $\mathrm{DC}(\mathrm{mm})$ \\
\hline \multirow{3}{*}{$1696,20 \mathrm{~mm}$} & \multirow{3}{*}{$27,40 \mathrm{a}$} & RB962962 & $27,76 \mathrm{ab}$ & $70 \mathrm{~N}+63 \mathrm{~K}$ & $27,24 a b$ \\
\hline & & RB867515 & $28,24 \mathrm{a}$ & $140 \mathrm{~N}+126 \mathrm{~K}$ & $27,24 \mathrm{ab}$ \\
\hline & & RB92579 & $26,99 \mathrm{ab}$ & $210 \mathrm{~N}+189 \mathrm{~K}$ & $27,95 \mathrm{a}$ \\
\hline \multirow{2}{*}{$1935,21 \mathrm{~mm}$} & \multirow{2}{*}{$27,11 \mathrm{a}$} & RB863129 & $27,28 \mathrm{ab}$ & $280 \mathrm{~N}+252 \mathrm{~K}$ & $26,61 \mathrm{~b}$ \\
\hline & & RB98710 & $26,02 \mathrm{~b}$ & & \\
\hline
\end{tabular}

Nas colunas, médias seguidas da mesma letra não diferem estatisticamente ao nível de $5 \%$ pelo teste Tukey. $\mathrm{N}=$ Nitrogênio; $\mathrm{K}=$ Potássio

Isoladamente, ocorreram diferenças entre variedades $(\mathrm{P}<0,05)$, sendo que a variedade 
RB867515 exibiu a maior altura de plantas $(4,66$ $\mathrm{m})$, maior tamanho $(3,56 \mathrm{~m})$ e diâmetro $(28,24$ $\mathrm{cm}$ ) dos colmos em relação aos demais materiais testados. Já a variedade RB98710 apresentou menor altura $(4,15 \mathrm{~m})$, menor tamanho $(3,08 \mathrm{~m}) \mathrm{e}$ menor diâmetro do colmo $(26,02 \mathrm{~cm})$, enquanto as variedades RB92579 e RB863129 ficaram em posição intermediária. Ressalta-se que o colmo possui as funções de armazenamento de sólidos solúveis e de sustentação da planta.

A variedade RB867515, também, sobressaiu em tamanho e diâmetro do colmo nos trabalhos conduzidos por Marques and Silva (2008) e Bezerra et al. (2017). Nas condições edafoclimáticas de Salinas, MG, avaliando cinco variedades de cana-de-açúcar, Barbosa (2005) aferiu, no momento da colheita, alturas variando entre 4,09 $\mathrm{m}$ e 4,60 m e diâmetros do colmo de $3,10 \mathrm{~cm}$ a $3,72 \mathrm{~cm}$. Oliveira et al. (2010) testando onze variedades de cana-de-açúcar, revelaram maiores crescimentos do colmo para as variedades RB92579 (3,11 m) e RB867515 (3,04 $\mathrm{m})$.

As aplicações combinadas de nitrogênio e potássios testadas não influenciaram a altura de plantas nem o tamanho do colmo, contudo, a dose $210 \mathrm{~N}+189 \mathrm{~K} / \mathrm{ha}$ resultou em maior diâmetro do colmo que, estatisticamente, foi superior à aplicação de $280 \mathrm{~N}+252 \mathrm{~K} / \mathrm{ha}$, e igualáveis às demais doses testadas (Tabela 1). O incremento do diâmetro do colmo promovido pela adubação constitui um fator importante do ponto de vista morfofisiológico da cana-deaçúcar, devido à probabilidade de redução do acamamento, fato frequente em Poaceas de grande porte e de rápido crescimento. A altura de plantas, o tamanho e o diâmetro do colmo são variáveis com forte componente genético, mas sujeitas a influências ambientais e com relação direta com a produção da cana-de-açúcar.

\section{Parâmetros produtivos}

Não foi observada interação $(P>0,05)$ entre as lâminas de irrigação, variedades e níveis de adubação sobre os parâmetros de produção (produtividade total de matéria verde - PTMV, produtividade total de matéria seca - PTMS, produtividade de matéria verde de colmos PMVC, e porcentagem de colmos - \%C). Isoladamente as lâminas de irrigação promoveram efeitos estatísticos $(\mathrm{P}<0,05)$ sobre a PTMS, cuja menor lâmina produziu 63,25 t/ha, isto é, 9,8\% inferior a maior lâmina de irrigação (70,16 t de MS/ha) (Tabela 2). Contudo, as lâminas de irrigação não apresentaram diferenças significativas sobre a produção de biomassa, demonstrando ser possível produzir no mínimo 242,60 t/ha matéria verde de cana-de-açúcar ou 63,25 t de matéria seca, da qual 83,94\% correspondem à fração colmo. Em Paracatu, Minas Gerais, Oliveira et al. (2002) reportaram que sob irrigação e adubação, sete variedades de cana-de-açúcar produziram de 175 a 230 t/ha de matéria verde, e de 137 a 184 t/ha de colmos. Em Presidente Médici, Rondônia, região cuja precipitação pluvial varia entre 2.000 e 2.500 $\mathrm{mm}$, Townsend et al. (2012) registraram produtividades médias de 56 e 223 t/ha, respectivamente, de matéria seca e matéria verde, em 11 variedades de cana-de-açúcar.

Ocorreram diferenças significativas entre as variedades sobre PTMV, PTMS e PMVC (Tabela 2). As variedades mais produtivas em termos de biomassa verde (planta inteira e colmo) foram a RB962962 e a RB867515. A variedade RB962962 também apresentou maior produção de matéria seca. Nas condições edafoclimáticas de Teresina, PI, durante três ciclos de cultivo, as variedades RB962962 (162,3 t/ha) e RB867515 $(158,5 \mathrm{t} / \mathrm{ha})$ apresentam maior produtividade de colmos em comparação às outras variedades, em vários níveis de lâminas de irrigação. Ressalta-se que no primeiro ciclo a RB867515 produziu 210,9 t/ha, $24,7 \%$ a mais que as demais variedades testadas (Andrade Junior et al. 2017). É importante enfatizar que a cana-de-açúcar é uma espécie altamente eficiente em conversão energética, uma vez que seu metabolismo fotossintético é do tipo $\mathrm{C} 4$, caracterizado pela eficiência na fixação de $\mathrm{CO}_{2}$. Além disso, em condições de alta luminosidade e disponibilidade de água, a espécie pode apresentar elevada produção de biomassa (Oliveira et al. 2015).

Os níveis de adubação não influenciaram $(\mathrm{P}$ > 0,05 ) os parâmetros de produtividade (Tabela 2). Isso permite inferir que as condições edáficas locais, onde predominam solos arenosos, podem ser limitantes à utilização do complexo irrigação + adubação (NK), pois, conforme Lawton et al. (1978) há grande probabilidade de perdas de água por percolação e lixiviação de nutrientes. 
Tabela 2. Respostas da produtividade total de matéria verde (PTMV), produtividade total de matéria seca (PTMS), produtividade de matéria verde de colmos (PMVC), porcentagem de colmos (\%C) da cana-de-açúcar aos efeitos isolados das lâminas de irrigação, variedades e adubação. Parnaíba-PI, 2016.

\begin{tabular}{|c|c|c|c|c|c|}
\hline Laminas de água de irrigação (mm) & PTMV (t/ha) & Variedades cana-de-açúcar & PTMV (tha) & Adubação (k/ha) & PTMV (tha) \\
\hline \multirow{3}{*}{$1696,20 \mathrm{~mm}$} & \multirow{3}{*}{$242,60 \mathrm{a}$} & RB962962 & $289,44 \mathrm{a}$ & $70 \mathrm{~N}+63 \mathrm{~K}$ & $237,84 \mathrm{a}$ \\
\hline & & RB867515 & $267,55 \mathrm{a}$ & $140 \mathrm{~N}+126 \mathrm{~K}$ & $241,72 \mathrm{a}$ \\
\hline & & RB92579 & $255,29 a b$ & $210 N+189 K$ & $276,86 \mathrm{a}$ \\
\hline \multirow{3}{*}{$1935,21 \mathrm{~mm}$} & \multirow{2}{*}{$262,76 \mathrm{a}$} & RB863129 & $242,20 \mathrm{ab}$ & $280 \mathrm{~N}+252 \mathrm{~K}$ & $254,29 \mathrm{a}$ \\
\hline & & RB98710 & $208,24 b$ & & \\
\hline & PTMS (t/ha) & & PTMS (t/ha) & & PTMS (t/ha) \\
\hline \multirow{3}{*}{$1696,20 \mathrm{~mm}$} & \multirow{3}{*}{$63,25 \mathrm{~b}$} & RB962962 & $77,35 \mathrm{a}$ & $70 \mathrm{~N}+63 \mathrm{~K}$ & $62,52 \mathrm{a}$ \\
\hline & & RB867515 & $70,18 \mathrm{ab}$ & $140 N+126 K$ & $63,90 \mathrm{a}$ \\
\hline & & RB92579 & $66,91 \mathrm{ab}$ & $210 N+189 K$ & $73,45 \mathrm{a}$ \\
\hline \multirow{3}{*}{$1935,21 \mathrm{~mm}$} & \multirow{2}{*}{70,16 a } & RB863129 & $61,89 \mathrm{~b}$ & $280 \mathrm{~N}+252 \mathrm{~K}$ & $66,93 \mathrm{a}$ \\
\hline & & RB98710 & $57,18 \mathrm{~b}$ & & \\
\hline & $\operatorname{PMVC}(\mathrm{t} / \mathrm{ha})$ & & PMVC (t/ha) & & $\operatorname{PMVC}(\mathrm{t} / \mathrm{ha})$ \\
\hline \multirow{3}{*}{$1696,20 \mathrm{~mm}$} & \multirow{3}{*}{$203,77 \mathrm{a}$} & RB962962 & $246,93 \mathrm{a}$ & $70 \mathrm{~N}+63 \mathrm{~K}$ & $201,65 \mathrm{a}$ \\
\hline & & RB867515 & $225,50 \mathrm{a}$ & $140 N+126 K$ & $235,57 \mathrm{a}$ \\
\hline & & RB92579 & $218,12 a b$ & $210 N+189 K$ & $235,96 \mathrm{a}$ \\
\hline \multirow{3}{*}{$1935,21 \mathrm{~mm}$} & \multirow{2}{*}{$225,75 \mathrm{a}$} & RB863129 & $206,35 a b$ & $280 \mathrm{~N}+252 \mathrm{~K}$ & $215,87 \mathrm{a}$ \\
\hline & & RB98710 & $176,91 \mathrm{~b}$ & & \\
\hline & $\% \mathrm{C}$ & & $\% \mathrm{C}$ & & $\% \mathrm{C}$ \\
\hline \multirow{3}{*}{$1696,20 \mathrm{~mm}$} & \multirow{3}{*}{83,87 a } & RB962962 & $85,22 \mathrm{a}$ & $70 \mathrm{~N}+63 \mathrm{~K}$ & $85,11 \mathrm{a}$ \\
\hline & & RB867515 & $84,51 \mathrm{a}$ & $140 N+126 K$ & $85,24 \mathrm{a}$ \\
\hline & & RB92579 & $85,63 \mathrm{a}$ & $210 N+189 K$ & $84,13 \mathrm{a}$ \\
\hline \multirow{2}{*}{$1935,21 \mathrm{~mm}$} & \multirow{2}{*}{$86,22 \mathrm{a}$} & RB863129 & $84,80 \mathrm{a}$ & $280 \mathrm{~N}+252 \mathrm{~K}$ & 84,70 a \\
\hline & & RB98710 & 85,07 a & & \\
\hline
\end{tabular}

Nas colunas, médias seguidas da mesma letra não diferem estatisticamente ao nível de 5\% pelo teste Tukey. $\mathrm{N}=$ Nitrogênio; $\mathrm{K}=$ Potássio

Considerando que não houve resposta significativa dos parâmetros de produtividade (PTMV, PTMS, PMVC e \%C) aos níveis de adubação testados, sugere-se a adoção da menor lâmina de irrigação e menor dose de adubação $(70 N+63 K)$, em função da economia de água e fertilizantes. É importante destacar que neste experimento todas as variedades tiveram a participação de colmos superior a $80 \%$, um dos principais atributos qualitativos destacados por Rodrigues et al. (1997), na seleção de variedades de cana-de-açúcar para alimentação de ruminantes.

\section{Parâmetros nutricionais}

Não foi observada interação $(P>0,05)$ entre as lâminas de irrigação, variedades e níveis de adubação sobre os parâmetros nutricionais: $\mathrm{PB}$, FDN, FDA, Brix e a razão FDN/Brix da cana-deaçúcar (Tabela 3).

As lâminas de irrigação, as variedades e os níveis adubação não promoveram efeitos $(\mathrm{P}<$ 0,05 ) sobre os teores de $\mathrm{PB}$, cuja média geral foi $2,19 \%$ (Tabela 3). As médias observadas estão de acordo com as descritas por Pinto et al. (2003), que após revisarem vários trabalhos sobre as características nutricionais da cana-de-açúcar, reportaram teores de PB entre 1,7\% e 3,2\%, com média de 2,4\%. Em Goiânia, Goiás, Mello et al. (2006) obtiveram média $1,89 \%$ a $3,20 \%$ em nove variedades mantidas sob irrigação por aspersão. Contudo, considerando que o nível mínimo de proteína nos alimentos deve ser de $7 \%$ para que ocorra adequada fermentação ruminal (Minson 2012), os tratamentos aqui testados estão aquém deste limite. Ressalta-se que os baixos níveis de PB é uma característica própria da cana-deaçúcar (López et al. 2003).

A FDN é constituída por substâncias da parede celular, basicamente, celulose, hemicelulose, lignina e proteína lignificada. Os maiores teores $(\mathrm{P}<0,05)$ de FDN foram encontrados nas plantas que receberam a menor lâmina de irrigação, $45,71 \%$, contra $43,23 \%$ da maior lâmina de irrigação. Estes resultados são importantes, pois teores de FDN elevados limitam a ingestão de cana-de-açúcar e, consequentemente, o consumo de energia será insuficiente para atender às exigências nutricionais do animal, afetando o seu desempenho. 
Tabela 3. Respostas dos teores de proteína bruta (\%PB), Brix (\%), fibra em detergente ácido (FDA), fibra em detergente neutro (FDN) e relação FDN/Brix da planta inteira da cana-de-açúcar aos efeitos isolados das lâminas de irrigação, variedades e adubação. Parnaíba-PI, 2016.

\begin{tabular}{|c|c|c|c|c|c|}
\hline $\begin{array}{c}\text { Laminas de água de irrigação } \\
(\mathrm{mm})\end{array}$ & PB $(\%)$ & $\begin{array}{l}\text { Variedades cana-de- } \\
\text { açúcar }\end{array}$ & $\mathrm{PB}(\%)$ & $\begin{array}{c}\text { Adubação } \\
(\mathrm{k} / \mathrm{ha})\end{array}$ & $\mathrm{PB}(\%)$ \\
\hline \multirow{3}{*}{$1696,20 \mathrm{~mm}$} & \multirow{2}{*}{$2,27 \mathrm{a}$} & RB962962 & $2,16 \mathrm{a}$ & $70 N+63 K$ & $2,12 \mathrm{a}$ \\
\hline & & RB867515 & $2,08 \mathrm{a}$ & $140 \mathrm{~N}+126 \mathrm{~K}$ & $2,19 \mathrm{a}$ \\
\hline & \multirow{3}{*}{$2,12 \mathrm{a}$} & RB92579 & $2,28 \mathrm{a}$ & $210 N+189 K$ & $2,17 \mathrm{a}$ \\
\hline \multirow{3}{*}{$1935,21 \mathrm{~mm}$} & & RB863129 & $2,32 \mathrm{a}$ & $280 \mathrm{~N}+252 \mathrm{~K}$ & $2,30 \mathrm{a}$ \\
\hline & & RB98710 & $2,13 \mathrm{a}$ & & \\
\hline & FDN (\%) & & FDN (\%) & & FDN $(\%)$ \\
\hline \multirow{3}{*}{$1696,20 \mathrm{~mm}$} & \multirow{3}{*}{$45,71 \mathrm{a}$} & RB962962 & $47,03 \mathrm{a}$ & $70 \mathrm{~N}+63 \mathrm{~K}$ & $43,49 \mathrm{a}$ \\
\hline & & RB867515 & $44,17 \mathrm{~b}$ & $140 \mathrm{~N}+126 \mathrm{~K}$ & $45,38 \mathrm{a}$ \\
\hline & & RB92579 & $44,58 \mathrm{ab}$ & $210 \mathrm{~N}+189 \mathrm{~K}$ & $44,14 \mathrm{a}$ \\
\hline \multirow{2}{*}{$1935,21 \mathrm{~mm}$} & \multirow{2}{*}{$43,23 \mathrm{~b}$} & RB863129 & $43,79 \mathrm{~b}$ & $280 \mathrm{~N}+252 \mathrm{~K}$ & $44,86 \mathrm{a}$ \\
\hline & & RB98710 & $42,77 \mathrm{~b}$ & & \\
\hline & Brix $(\%)$ & & Brix (\%) & & Brix $(\%)$ \\
\hline \multirow{3}{*}{$1696,20 \mathrm{~mm}$} & \multirow{3}{*}{$18,41 \mathrm{a}$} & RB962962 & $18,22 \mathrm{ab}$ & $70 \mathrm{~N}+63 \mathrm{~K}$ & $18,60 \mathrm{a}$ \\
\hline & & RB867515 & $18,93 \mathrm{a}$ & $140 \mathrm{~N}+126 \mathrm{~K}$ & $18,50 \mathrm{a}$ \\
\hline & & RB92579 & $18,94 \mathrm{a}$ & $210 N+189 K$ & $18,97 \mathrm{a}$ \\
\hline \multirow{3}{*}{$1935,21 \mathrm{~mm}$} & \multirow{2}{*}{$18,73 \mathrm{a}$} & RB863129 & $17,84 \mathrm{~b}$ & $280 \mathrm{~N}+252 \mathrm{~K}$ & $18,19 \mathrm{a}$ \\
\hline & & RB98710 & $18,92 \mathrm{a}$ & & \\
\hline & FDN/Brix & & FDN/Brix & & FDN/Brix \\
\hline \multirow{3}{*}{$1696,20 \mathrm{~mm}$} & \multirow{3}{*}{$2,50 \mathrm{a}$} & RB962962 & $2,59 \mathrm{a}$ & $70 \mathrm{~N}+63 \mathrm{~K}$ & $2,37 \mathrm{a}$ \\
\hline & & RB867515 & $2,35 \mathrm{bc}$ & $140 \mathrm{~N}+126 \mathrm{~K}$ & $2,47 \mathrm{a}$ \\
\hline & & RB92579 & $2,37 \mathrm{bc}$ & $210 \mathrm{~N}+189 \mathrm{~K}$ & $2,34 \mathrm{a}$ \\
\hline \multirow{3}{*}{$1935,21 \mathrm{~mm}$} & \multirow{2}{*}{$2,32 \mathrm{a}$} & RB863129 & $2,47 \mathrm{ab}$ & $280 \mathrm{~N}+252 \mathrm{~K}$ & $2,48 \mathrm{a}$ \\
\hline & & RB98710 & $2,28 \mathrm{c}$ & & \\
\hline & FDA (\%) & & FDA (\%) & & FDA (\%) \\
\hline \multirow{3}{*}{$1696,20 \mathrm{~mm}$} & \multirow{3}{*}{28,29 a } & RB962962 & $27,82 \mathrm{a}$ & $70 N+63 K$ & $27,73 \mathrm{a}$ \\
\hline & & RB867515 & 27,99 a & $140 \mathrm{~N}+126 \mathrm{~K}$ & $28,32 \mathrm{a}$ \\
\hline & & RB92579 & $27,60 \mathrm{a}$ & $210 N+189 K$ & 27,99 a \\
\hline \multirow[b]{2}{*}{$1935,21 \mathrm{~mm}$} & \multirow[b]{2}{*}{$27,64 \mathrm{a}$} & RB863129 & $28,97 \mathrm{a}$ & $280 \mathrm{~N}+252 \mathrm{~K}$ & $27,81 \mathrm{a}$ \\
\hline & & RB98710 & $27,41 \mathrm{a}$ & & \\
\hline
\end{tabular}

Nas colunas, médias seguidas da mesma letra não diferem estatisticamente ao nível de 5\% pelo teste Tukey. N=Nitrogênio; $\mathrm{K}=$ Potássio

Ocorreram diferenças significativas entre as variedades de cana-de-açúcar em relação aos teores de FDN (Tabela 3), cujos maiores valores foram observados na variedade RB962962 $(47,03 \%)$, que foi semelhante à variedade RB92579 (44,58\%), seguida das variedades RB867515 (44,17\%), RB863129 (43,79\%) e RB98710 (42,77\%). Com média de $44,6 \%$, os teores de FDN não responderam às adubações testadas. Ressalta-se que o teor de FDN da canade-açúcar é influenciado, principalmente, pelas variedades e pela idade de corte.

Em geral, excetuando a média apresentada pela variedade RB962962, os teores de FDN aqui constatados são inferiores aos reportados por Rodrigues et al. (1997), cujas médias variaram de 45,19 a $56,47 \%$ em 11 cultivares de cana-deaçúcar, irrigadas e adubadas. É importante reportar que teores de FDN menores que 50\%, poderão incrementar o consumo da cana-deaçúcar por duas razões básicas: o baixo teor de
FDN na planta corresponde ao maior teor de nutrientes do conteúdo celular, que são de rápida digestão, e, um baixo teor de FDN significa também que a celulose e a hemicelulose da parede celular apresentam taxa de fermentação mais rápida no rúmen, devido à sua menor lignificação. A maior taxa de fermentação da fibra vegetal contribui decisivamente para diminuir a repleção ruminal, o que estimula o aumento do consumo pelo animal e, consequentemente, sua produção.

Quanto aos teores de sólidos solúveis (Brix), apenas as variedades responderam aos tratamentos testados (Tabela 3). Os maiores percentuais foram observados nas variedades RB922579 (18,94\%), RB867515 (18,93\%) e RB9810 (18,92\%), enquanto o menor valor foi registrado na variedade RB863129 (17,84\%). Estes resultados estão próximos aos relatados por Bezerra (2014), que após conduzir experimento com variedades de cana-de-açúcar irrigadas e 
adubadas, encontraram Brix de 19,01\% (RB867515), 18,63\% (RB98710), 18,39\% (RB92579) e 17,30\% (RB863129). Em Anadia, Alagoas, após avaliarem o comportamento da cana-de-açúcar no ciclo de cana-planta (14 meses), Silva et al. (2013) reportaram que as variedades RB922579 e RB867515 apresentaram Brix de $17,31 \%$ e $17,35 \%$, respectivamente. Ressalta-se que as citadas variedades foram adubadas com 60, 100 e $150 \mathrm{~kg} / \mathrm{ha}$ de $\mathrm{N}, \mathrm{P}_{2} \mathrm{O}_{5}$ e $\mathrm{K}_{2} \mathrm{O}$, e que o volume de precipitação no período experimental foi de $1.873 \mathrm{~mm}$. Em Rio Largo, Alagoas, em canaviais adubados com NPK, Cruz et al. (2009) alcançaram teores de Brix de $20,94 \%$ e $21,98 \%$, respectivamente, para as variedades RB922579 e RB867515. Enquanto Lui et al. (2011) trabalhando com 13 variedades de cana-de-açúcar, irrigadas e adubadas, encontraram teores de Brix variando entre $18,33 \%$ e $23,67 \%$.

Os teores de Brix correspondem aos teores de sólidos solúveis presentes no colmo e está correlacionado com o teor de sacarose, e que, o ponto ideal de colheita da cana-de-açúcar, tanto para indústria, como para alimentação animal, é alcançado no período seco, momento que a planta atinge a maturação (Aude et al. 1992; Lui et al. 2011). Preston (1977) sugere que o teor adequado para o Brix da cana-de-açúcar utilizada na engorda de bovinos seja maior que $12 \%$, valor superado por todos os tratamentos aqui avaliados (Tabela 3).

As lâminas de irrigação e os níveis de adubação aplicados não influenciaram $(\mathrm{P}>0,05)$ a razão FDN/Brix, no entanto ocorreram diferenças significativas entre as variedades, cuja maior e a menor relação foram apresentadas pelas variedades RB962962 (2,59) e RB98710 $(2,28)$, respectivamente. Segundo Rodrigues et al. (1997) para não afetar o consumo da cana-de-açúcar por ruminantes, aceita-se como média 2,7 a razão FDN/Brix. Neste trabalho, todas as variáveis testadas se aproximam deste valor, independentemente das lâminas de irrigação, das variedades e dos níveis de adubação.

Com média de $27,96 \%$, os teores de FDA não responderam aos tratamentos impostos. Todavia, estão próximos dos valores adequados para a maioria das plantas forrageiras que oscilam em $30 \%$, por favorecerem a digestibilidade da forragem ingerida (Nussio et al. 1998). A FDA é constituída de celulose, lignina, além de quantidades variáveis de cinza e compostos nitrogenados indigestíveis (Bianchini et al. 2007). A FDA representa a quantidade de fibra que não é digerível, além de ser um indicador do valor energético do alimento (Rodrigues 2010).

\section{Conclusões}

As variedades de cana-de-açúcar apresentaram produtividades superiores a 200 t/ha, das quais, mais de $80 \%$ corresponderam aos colmos, demonstrando viabilidade técnica de serem utilizadas para alimentação animal.

As variedades RB962962 e RB867515 se sobressaíram pela elevada produtividade de biomassa e maiores percentuais de colmos, conciliada a satisfatórios teores de sólidos solúveis (açúcares).

A lâmina de irrigação de 1696,2 mm, associada à aplicação de $70 \mathrm{~kg}$ de N/ha/ano e 63 $\mathrm{kg}$ de $\mathrm{K}_{2} \mathrm{O} / \mathrm{h}$ /ano pode ser utilizada na produção de biomassa da cana-de-açúcar com fins forrageiros.

\section{Referências bibliográficas}

Andrade A.P., Costa R.G., Santos E.M. \& Silva D.S. 2010. Produção animal no semiárido: o desafio de disponibilizar forragem, em quantidade e com qualidade, na estação seca. Tecnologia \& Ciência Agropecuária 4, 1-14.

Andrade J.B., Ferrari Júnior E., Possenti R.A., Otsuki I.P., Zimback L. \& Landell M.G.A. 2004. Composição química de genótipos de cana-de-açúcar rm duas idades, para fins de nutrição animal. Bragantia 63, 341-349.

Andrade Junior A.S., Bastos E.A., Ribeiro V.Q., Athayde Sobrinho C. \& Silva P.H.S. 2017. Stalk yield of sugarcane cultivars under different water regimes by subsurface drip irrigation. Revista Brasileira de Engenharia Agricola e Ambiental 21, 169-174.

ANUALPEC 2018. Anuário da Pecuária Brasileira. Instituto FNP, São Paulo, SP, Brasil.

Aude M.I.S., Marchezan E. \& Pasqualetto A. 1992. Época de plantio e seus efeitos na produtividade e teor de sólidos solúveis no caldo da cana-de-açúcar. Ciência Rural 22, 131-137.

Barbosa E.A. 2005. Avaliação fitotécnica de cinco variedades de cana-de-açúcar para o município de Salinas-MG. 70 p. Universidade Estadual do Sudoeste da Bahia, Vitória da Conquista, Bahia, Brasil. 
Bezerra J.D.C. 2014. Competição de variedades de cana-de-açúcar para forragem no agreste de Pernambuco. In: Pós Graduação em Ciência Animal e Pastagens, 98 p. Universidade Rural de Pernambuco, Garanhuns, Garanhuns, Pernambuco, Brasil.

Bezerra J.D.C., Ferreira G.D.G., Campos J.M.S., Oliveira M.W., Andrade A.P. \& Nascimento Júnior J.R.S. 2017. Biometric and chemical characteristics of sugarcane varieties for use as forage in limiting soil water conditions. Revista Brasileira de Zootecnia 46, 384-392.

Bianchini W., Rodrigues É., Jorge A.M. \& Andrigheto C. 2007. Importância da fibra na nutrição de bovinos. REDVET. Revista Electrónica de Veterinaria 8, 1-14.

Bonomo P., Cardoso C.M.M., Pedreira M.S., Santos C.C., Pires A.J.V. \& Silva F.F. 2009. Potencial forrageiro de variedades de cana-deaçúcar para alimentação de ruminantes. Acta Scientiarum. Animal Sciences 31, 54-59.

Braga R.A.P., Cabral J.J.S.P., Montenegro S.M.G.L. \& Perrier Júnior G.S. 2002. Conservação dos recursos hídricos em brejos de altitude-O caso de Brejo dos Cavalos, Caruaru, PE. Revista Brasileira de Engenharia Agricola e Ambiental 6, 539-546.

Cândido M.J.D., Araújo G.G.L. de \& Cavalcante M.A.B. 2005. Pastagens no ecossistema semiárido brasileiro: atualização e perspectivas futuras. In: Reunião da Sociedade Brasileira de Zootecnia, 42., 2005, Goiânia, GO. Anais... Goiânia: SBZ, UFG, p.85-94.

Carlos Filho F., Távora F.J.A.F. \& Cruz H.L.L. 1986. Avaliação agroindustrial de variedades de cana-de-açúcar na região do Cariri Cearense. Pesquisa Agropecuária Brasileira 21, 155-163.

Carvalho M.V., Rodrigues P.H.M., Lima M.L.P., Anjos I.A., Landell M.G.A., Santos M.V. \& Silva L.F.P. 2010. Composição bromatológica e digestibilidade de cana-de-açúcar colhida em duas épocas do ano. Brazilian Journal of Veterinary Research and Animal Science 47, 298-306.

Cintra J.E.V., Ferreira G.H. \& Brasil R.P.C. 2008. Viabilidade da irrigação suplementar na fase inicial de desenvolvimento da cana-deaçúcar (Saccharum ssp.) em regiões com déficit hídrico. Nucleus 1, 1-9.

Cruz S.J.S., Oliveira S.S.C., Cruz S.C.S., Machado C.G. \& Pereira R.G. 2009. Efeito da adubação fosfata sobre o acúmulo de biomassa e teor de brix de duas variedades de cana-de-açúcar. Revista Caatinga 22, 110116.

Dalri A.B. \& Cruz R.L. 2008. Produtividade da cana-de-açúcar fertirrigada com $\mathrm{N}$ e $\mathrm{K}$ via gotejamento subsuperficial. Engenharia Agrícola 28, 516-524.

Doorembos J. \& Kassam A.H. 1979. Efeito da água no rendimento das culturas. Estudos FAO Irrigação e Drenagem 33, 42-53.

Fernandes A.M., Queiroz A.C., Pereira J.C., Lana R.P., Barbosa M., Fonseca D.M., Detmann E., Cabral L.S., Pereira E.S. \& Vittori A. 2003. Composição químico-bromatológica de variedades de cana-de-açúcar (Saccharum spp. L.) com diferentes ciclos de produção (precoce e intermediário) em três idades de corte. Revista Brasileira de Zootecnia 32, 977-985.

Freitas A.W.P., Rocha F.C., Zonta A., Fagundes J.L., Fonseca R. \& Zonta M.C.M. 2011. Desempenho de novilhos recebendo dietas à base de cana-de-açúcar in natura ou hidrolisada. Revista Brasileira de Zootecnia 40, 2532-2537.

Köppen W. \& Geiger R. 1928. Klimate der Erde. Gotha: Verlag Justus Perthes. Wall-map $150 \mathrm{~cm} \times 200 \mathrm{~cm}$.

Landell M.G.A., Campana M.P., Rodrigues A.A., Figueiredo P., Silva M.A., Bidoia, M.A.P. Rossetor, R. \& Martins A.L.M. 2002. A variedade IAC 86-2480 como nova opção de cana-de-açúcar para fins forrageiros: manejo de produção e uso na alimentação animal. Campinas: IAC, 36 p. (Boletim Técnico, 193).

Lawton K., Coelho M.A. \& Crisóstomo L.A. 1978. Movimento e perdas por lixiviacao de nutrientes so luveis aplicados a solos do Estado do Ceara, Brasil. Ciência agronômica 8, 9-18.

López I., Aranda E.M., Ramos J.A. \& Mendoza G.D. 2003. Evaluación nutricional de ocho variedades de caña de azúcar con potencial forrajero. Revista Cubana de Ciencia Agrícola 37, 381-386.

Lui J.J., Fidelis R.R., Dias M.A.R. \& Sarmento R.A. 2011. Produtividade de rapadura de genótipos de cana-de-açúcar na Região de Dueré, Sul de Tocantins. Semina: Ciências Agrárias 32, 1059-1068.

Marques T.A. \& Silva W.H. 2008. Crescimento vegetativo e maturação em três cultivares de 
cana-de-açúcar. Revista de Biologia $e$ Ciências da Terra 8, 54-60.

Mello S.Q.S., França A.F.S., Lima M.L.M., Ribeiro D.S., Miyagi E.S. \& Reis J.G. 2006. Parâmetros do valor nutritivo de nove variedades de cana-de-açúcar cultivadas sob irrigação. Ciência Animal Brasileira 7, 373380.

Melo F.B., Cavalcante A.C., Andrade Júnior A.S. $\&$ Bastos E.A. 2004. Levantamento detalhado dos solos da área da Embrapa MeioNorte/UEP de Parnaíba. Teresina: Embrapa Meio-Norte 89, 26p.

Minson D. 2012. Forage in ruminant nutrition. Academic Press, New York. 483p.

Nunes Filho J., Sá V.A.L., Sousa A.R., Ferraz L.G.B., Tabosa J.N., Santos V.F. \& Silva A.B. 2007. Gramíneas forrageiras tropicais em solo slino-sódico, sob irrigação, no Vale do Rio Moxotó-Pernambuco. Embrapa Solos-Artigo 14, 19-24.

Nussio L.G., Manzano R.P. \& Pedreira C.G.S. 1998. Valor alimentício em plantas do gênero Cynodon. In: Simpósio sobre Manejo da Pastagem, pp. 203-242. FEALQ/ESALQ, Piracicaba.

Oliveira A.R., Braga M.B. \& Walker A.M. 2015. Comportamento vegetativo e qualidade tecnológica de cultivares de cana-de-açúcar submetidas ao estresse hídrico em condições Semiáridas do Brasil. Revista Brasileira de Geografia Física 8, 525-541.

Oliveira E.C.A., Oliveira R.L., Andrade B.M.T., Freire F.J., Lira Júnior M.A. \& Machado P.R. 2010. Crescimento e acúmulo de matéria seca em variedades de cana-de-açúcar cultivadas sob irrigação plena. Revista Brasileira de Engenharia Agricola e Ambiental 14, 951960.

Oliveira M.W., Mendes L.C., Barbosa M.H.P., Vitti A.C. \& Faria R.O. 2002. Avaliação de sete variedades de cana-de-açúcar sob irrigação complementar. Reunião Brasileira de Fertilidade do Solo e Nutrição de Plantas 101, 9-10.

Pigden W.J. 1974. La caña de azucar descortezada como pienso: un paso decisivo. Revista Mundial de Zootecnia, 11, 1-5.

Pinto A.P., Pereira E.S. \& Mizubuti I.Y. 2003. Características nutricionais e formas de utilização da cana-de-açúcar na alimentação de ruminantes. Semina: Ciências Agrárias 24, 73-84.
Preston T.R. 1977. Nutritive value of sugar cane for ruminants. Tropical Animal Production 2, 125-142.

Rodrigues R.C. 2010. Métodos de análises bromatológicas de alimentos: métodos físicos, químicos e bromatológicos. Pelotas: Embrapa Clima Temperado. 177p. Documentos, 306.

Rodrigues A.A., Primavesi O. \& Esteves S.N. 1997. Efeito da qualidade de variedades de cana-de-açúcar sobre seu valor como alimento para bovinos. Pesquisa Agropecuária Brasileira 32, 1333-1338.

Santos D.H., Silva M.A., Tiritan C.S., Foloni J.S.S. \& Echer F.R. 2011. Qualidade tecnológica da cana-de-açúcar sob adubação com torta de filtro enriquecida com fosfato solúvel. Revista Brasileira de Engenharia Agricola e Ambiental 15, 443-449.

Silva D.J. \& Queiroz A.C. 2002. Análise de alimentos: métodos químicos e biológicos. Universdiade Federal de Viçosa, Viçosa, Minas Gerais, Brasil 305p.

Silva S.F.A. \& Azevedo C.A.V. 2016. The Assistat Software Version 7.7 and its use in the analysis of experimental data. African Journal of Agricultural Research 11, 37333740.

Silva V.S.G., Oliveira M.W., Santos C.E.R.E.S., Oliveira D.C., Tenório J.C. \& Reis R.M.S. 2013. Qualidade industrial de quatro variedades de cana-de-açúcar em três anos agrícolas. In: Jornada de Ensino, Pesquisa e Extensão. UFRPE, Recife, Pernambuco, Brasil.

Townsend C.R., Costa N.L., Pereira R.G.A., Silva Netto F.G., Magalhães J.A. \& Rodrigues B.H.N. 2012. Competição de variedades de cana-de-açúcar para a alimentação de ruminantes em Presidente Médici, Rondônia. PUBVET 6, Art. 1429-1435.

Voltolini T.V., Silva J.G., Silva W.E.L., Nascimento J.M.L., Queiroz M.A.Á. \& Oliveira A.R. 2012. Valor nutritivo de cultivares de cana-de-açúcar sob irrigação. Revista Brasileira de Saúde e Produção Animal 13, 894-901.

Recebido: 10 setembro, 2018.

Aprovado: 9 novembro, 2018.

Publicado: 27 dezembro, 2018.

Licenciamento: Este artigo é publicado na modalidade Acesso Aberto sob a licença Creative Commons Atribuição 4.0 (CC-BY 4.0), a qual permite uso irrestrito, distribuição, reprodução em qualquer meio, desde que o autor e a fonte sejam devidamente creditados. 